\title{
Impactos físicos, nutricionais, psicológicos, sociais e educacionais em jovens com Doença Renal Crônica em seu tratamento
}

Physical, nutritional, psychological, social and educational impacts on young people with Chronic Kidney Disease in their treatment

Impactos físicos, nutricionales, psicológicos, sociales y educativos en los jóvenes con Enfermedad Renal Crónica en su tratamiento

Centro Universitário UninovaFapi, Brasil E-mail: Jocyellefblima@hotmail.com

Janayna Maceno Alves

ORCID: https://orcid.org/0000-0002-9810-702X

Faculdade de Educação São Francisco, Brasil E-mail: nayna-alves@hotmail.com

Helainy de Carvalho Freitas ORCID: https://orcid.org/0000-0002-8160-6033

Faculdade de Educação São Francisco, Brasil E-mail: helainyhcf@hotmail.com

Anne Karynne da Silva Barbosa ORCID: https://orcid.org/0000-0002-5993-8453

Universidade Federal do Maranhão, Brasil E-mail: karynnenutri@gmail.com

\section{Resumo}

Segundo a Sociedade Brasileira de Nefrologia (SBN) o Brasil em 2013 teve 50.961 pacientes fazendo tratamento de hemodiálise por conta da DRC. Tais dados, revelam que 5,6\% são pacientes na faixa etária de 13 a 18 anos, apesar da 
quantidade não ser tão significativa, nota-se o desenvolvimento gradual de novos casos em crianças e adolescentes, o resultado dessa pesquisa serve de alerta para a sociedade. Trata-se de um artigo de revisão integrativa de literatura, utilizando os artigos dos anos 2011 a 2021 sendo excluídos teses, dissertações, monografias e textos não relevantes. A doença renal crônica é uma patologia em nível crítico, a qual, o tratamento dialítico é muito invasivo, gerando limitações físicas, nutricionais e o desequilíbrio emocional e psicológico.

Palavras-chave: Nefropatias; Rim; Ciências da nutrição.

\begin{abstract}
According to the Brazilian Society of Nephrology (SBN) in 2013 Brazil had 50,961 patients undergoing hemodialysis treatment due to CKD. Such data reveal that 5.6\% are patients aged 13 to 18 years, although the number is not so significant, there is a gradual development of new cases in children and adolescents, the result of this research serves as a warning for the society. This is an integrative literature review article, using articles from the years 2011 to 2021 , excluding theses, dissertations, monographs and non-relevant texts. Chronic kidney disease is a pathology at a critical level, which dialysis treatment is very invasive, causing physical and nutritional limitations and emotional and psychological imbalance.
\end{abstract}

Keywords: Kidney diseases; Kidney; Nutritional sciences.

\title{
Resumen
}

Según la Sociedad Brasileña de Nefrología (SBN) en 2013 Brasil tenía 50.961 pacientes en tratamiento de hemodiálisis por ERC. la sociedad. Dichos datos revelan que el 5,6\% son pacientes de 13 a 18 años, aunque el número no es tan significativo, hay un desarrollo paulatino de nuevos casos en niños y adolescentes, el resultado de esta investigación sirve de advertencia. para la sociedad. Se trata de un artículo de revisión integradora de la literatura, utilizando artículos de los años 2011 a 2021, excluyendo tesis, disertaciones, monografías y textos no relevantes. La enfermedad renal crónica es una patología de nivel crítico, cuyo tratamiento de diálisis es muy invasivo, provocando limitaciones físicas y nutricionales y desequilibrio emocional y psicológico.

Palabras clave: Enfermedades renales; Riñón; Ciencias nutricionales.

\section{Introdução}

A doença renal crônica (DRC) é uma patologia de grande importância para a saúde pública, sendo relevante a sua pesquisa, visto que ela está fortemente associada com outras duas doenças crônicas não transmissíveis, tais como, diabetes mellitus e hipertensão arterial sistêmica (Mello, Moreira, 2016).

Esta anomalia altera gradualmente as funções renais, gerando o desequilíbrio em todo organismo, compromete outros órgãos e consequentemente promove o surgimento ou o agravamento de outras doenças (Resende et al., 2021).

Segundo a Sociedade Brasileira de Nefrologia (SBN) o Brasil em 2013 teve 50.961 pacientes fazendo tratamento de hemodiálise por conta da DRC. Tais dados, revelam que 5,6\% são pacientes na faixa etária de 13 a 18 anos, apesar da quantidade não ser tão significativa, nota-se o desenvolvimento gradual de novos casos em crianças e adolescentes, o resultado dessa pesquisa serve de alerta para a sociedade (Mello, Moreira, 2016).

Sabe-se que no intervalo entre o começo da adolescência e a fase adulta aparecem diversos desafios, a qual os jovens fazem grandes descobertas, sofrem mudanças sociais e psicológicas, passam a adaptar-se a novas realidades. No entanto, aqueles que são diagnosticados com patologias crônicas são obrigados a superar outras barreiras que comumente são associadas a pessoas idosas, uma consequência preocupante a este assunto é a crise psicossocial, dificultando a interação desses jovens a sociedade (Rêgo et al., 2019).

Crianças e adolescentes, sentem os impactos da Insuficiência Renal Crônica (IRC) com mais intensidade, pois, as limitações decorrentes das mudanças no cotidiano, da dieta e o tratamento desta doença afetam diretamente seu ponto de vista sobre a vida, gerando assim, medo, insegurança e alterações psicológicas (Abreu et al., 2015).

Nesse cenário pode-se destacar outro problema preocupante, o desempenho escolar. O tratamento de hemodiálise passa a ser prejudicial para o aprendizado dos estudantes, pois suas condições físicas e psicológicas não permitem que estes frequentem as escolas regularmente. Dessa forma, mesmo justificando suas faltas com documentos que comprovem as causas de suas ausências, continuam atrasados em relação aos conteúdos ministrados em sala de aula (Silva, Lemos, 2018). 
Diante dessa problemática, objetivou-se compreender os principais impactos físicos, nutricionais, psicológicos, sociais e educacionais em jovens que fazem tratamento de hemodiálise. Assim, a equipe de saúde juntamente com a família e gestores da educação podem proporcionar um atendimento mais humanizado, e como consequência positiva desse atendimento tem-se a qualidade de vida desses pacientes e a queda nos índices de atraso ou desistência escolar.

\section{Metodologia}

Trata-se de um artigo de revisão integrativa de literatura, com busca nas bases de dados, utilizando os termos: impactos na vida dos jovens com doença renal crônica, jovens e doença renal crônica, doença renal e impactos na escolaridade, utilizando os artigos dos anos 2011 a 2021 sendo excluídos teses, dissertações, monografias e textos não relevantes.

\section{Resultados e Discussão}

De acordo com o apresentado no quadro 1, estão dispostos os artigos selecionados após criteriosa busca sobre a temática em Doença Renal Crônica em jovens.

Quadro 1 - Artigos selecionados.

\begin{tabular}{|c|c|c|c|}
\hline Autor & Objetivo & Metodologia & Resultados \\
\hline $\begin{array}{l}\text { Mello, Moreira, } \\
\text { (2016) }\end{array}$ & $\begin{array}{l}\text { Analisar os jovens com doença } \\
\text { renal crônica (DRC) e sua } \\
\text { interação social. }\end{array}$ & $\begin{array}{c}\text { Pesquisa qualitativa feita em } \\
\text { instituição pública com foco na saúde } \\
\text { dos jovens, especificamente aqueles } \\
\text { com doença renal crônica baseado na } \\
\text { teoria da Sociologia Compreensiva de } \\
\text { Schutz. }\end{array}$ & $\begin{array}{l}\text { Destaca a importância das relações } \\
\text { simbólicas entre pacientes e } \\
\text { profissionais de saúde de forma } \\
\text { que contribua para o } \\
\text { desenvolvimento juvenil e estes } \\
\text { não sofram tantos com os impactos } \\
\text { sócias e psicológicos. }\end{array}$ \\
\hline Rêgo et al., (2019). & $\begin{array}{l}\text { Perceber sequelas sociais que } \\
\text { adolescentes encaram com o } \\
\text { diagnóstico que possuem doença } \\
\text { renal em estado final e seu } \\
\text { tratamento. }\end{array}$ & $\begin{array}{l}\text { Adolescentes de } 12 \text { a } 18 \text { anos } \\
\text { submeteram-se a um estudo } \\
\text { qualitativo e descritível, estes são } \\
\text { pacientes do Hospital da Criança José } \\
\text { Alencar- DF, no âmbito de Terapia } \\
\text { Renal Substitutiva (TRS) a qual tem } \\
\text { por finalidade aprofundar e } \\
\text { compreender este grupo. }\end{array}$ & $\begin{array}{c}\text { Constatou-se com essa pesquisa } \\
\text { que dentre os participantes pode } \\
\text { observar três categorias } \\
\text { modificadora da rotina: o } \\
\text { sentimento dos jovens em relação a } \\
\text { doença, o sentimento destes quanto } \\
\text { ao tratamento e a reação da família } \\
\text { a esta realidade. }\end{array}$ \\
\hline $\begin{array}{l}\text { Pennafort, Queiroz, } \\
\text { (2011) }\end{array}$ & $\begin{array}{l}\text { Discutir sobre os ele mentos } \\
\text { clínicos em relação as } \\
\text { necessidades das crianças e } \\
\text { jovens que possuem doença renal } \\
\text { crônica e seu tratamento. }\end{array}$ & $\begin{array}{l}\text { Através de estudo descritivo, a qual } \\
\text { foram avaliados } 33 \text { prontuários que } \\
\text { descreveram a identificação, a causa } \\
\text { da insuficiência renal crônica, peso, } \\
\text { comorbidades e tratamento. Este foi } \\
\text { realizado em uma clínica } \\
\text { especializada e vinculada ao SUS em } \\
\text { Fortaleza. }\end{array}$ & $\begin{array}{l}\text { Destaca-se o sexo feminino como } \\
\text { protagonista em (DRC), as } \\
\text { principais causas são as } \\
\text { glomerulopatias e uropatia, peso } \\
\text { maior ou igual a P3 e as } \\
\text { comorbidades mais frequentes são } \\
\text { hipertensão e cardiopatia. }\end{array}$ \\
\hline $\begin{array}{l}\text { Abreu et al., } \\
\text { (2015). }\end{array}$ & $\begin{array}{c}\text { Identificar em jovens e } \\
\text { adolescentes com Insuficiência } \\
\text { Renal Crônica (IRC) os impactos } \\
\text { do tratamento de hemodiálise na } \\
\text { qualidade de vida desses } \\
\text { pacientes e métodos que os } \\
\text { profissionais de saúde possam } \\
\text { melhorar essa expectativa. }\end{array}$ & $\begin{array}{l}\text { Estudo metodológico através de } \\
\text { coleta de dados em entrevista, } \\
\text { participaram desta pesquisa } 12 \\
\text { pessoas de } 2 \text { centros de dialise, } \\
\text { utilizou-se também o programa } \\
\text { MAXGDA para a análise do tema. }\end{array}$ & $\begin{array}{l}\text { Identificou-se sete temas } \\
\text { importante para o desenvolvimento } \\
\text { desses estudos, como o } \\
\text { autocuidado, apoio familiar e } \\
\text { evasão escolar. }\end{array}$ \\
\hline
\end{tabular}




\begin{tabular}{|c|c|c|c|}
\hline $\begin{array}{c}\text { Silva, Lemos, } \\
\text { (2018) }\end{array}$ & $\begin{array}{c}\text { Compreender os impactos do } \\
\text { tratamento da (DRC) na vida dos } \\
\text { jovens. }\end{array}$ & $\begin{array}{c}\text { Pesquisa qualitativa na Unidade de } \\
\text { Cuidados Renais do Hospital } \\
\text { Universitário- UFMA para } \\
\text { compreender a vivência dos }\end{array}$ & $\begin{array}{c}\text { É evidente que a hemodiálise, } \\
\text { tratamento para quem tem doença } \\
\text { renal crônica (DRC) afeta } \\
\text { diretamente a vida social dos } \\
\text { pacientes, considerando também, } \\
\text { jovens com esta enfermidade. }\end{array}$ \\
& $\begin{array}{c}\text { outros elementos como por exemplo, } \\
\text { a crença. }\end{array}$ & \\
\hline
\end{tabular}

Fonte: Autores.

Mello e Moreira (2016) em estudo com jovens de 12 a 20 anos com doença renal crônica (DRC) aborda a importância das relações afetiva da família e dos profissionais de saúde a estes adolescentes e o protagonismo juvenil a respeito da interação social, a fim de melhorar a expectava de vida de cada paciente.

Rêgo et al, (2019), em estudo, realizado em uma clínica que disponibiliza tratamento de hemodiálise para pessoas com Insuficiência Renal Crônica (IRC) através de pesquisa qualitativa com jovens de 12 a 18 anos destaca fatores importantes como os impactos sociais e emocionais nos adolescentes que passam por esse tratamento, o sentimento de medo e insegurança são frequentes.

Pennafort e Queiroz, (2019), em estudo baseado em análise de prontuários de crianças e adolescentes na situação de insuficiência renal crônica, foram discutidos como os profissionais de saúde podem estar melhorando o tratamento destes pacientes e atendendo suas necessidades. Destacando a importância dos profissionais de enfermagem serem capacitados para atuarem neste setor. Assim, estes poderão identificar quaisquer intercorrências e evitando o agravo ou sequelas no quadro do paciente.

Abreu et al, (2015), em estudo com crianças e adolescentes utilizando o método de entrevista, participaram 12 pacientes com doença crônica renal (DRC). Objetivou-se analisar aspectos consideráveis para a qualidade de vida, com esta pesquisar foram classificados alguns temas como base, dentre esses destacou-se a evasão escolar.

Silva e Lemos, (2018), seu estudo corrobora com os demais autores já citados, que objetiva compreender os principais impactos sofridos pelos jovens com doença renal crônica e a importância da família e dos profissionais de saúde no tratamento de hemodiálise para amenizar os desequilíbrios sociais, emocionais, psicológicos e a evasão escolar.

\section{Conclusão}

A doença renal crônica é uma patologia em nível crítico, a qual, o tratamento dialítico é muito invasivo, gerando limitações físicas, nutricionais e o desequilíbrio emocional e psicológico. No entanto, quando o diagnóstico é no jovem outros desafios são encontrados, principalmente em relação a qualidade de vida social. Por tanto, a partir da análise do crescimento de adolescentes em situação de insuficiência renal devem ser avaliados como casos especiais, para que estes sejam beneficiados com planos estratégicos, objetivando minimizar os impactos deste tratamento.

Ressalta-se ainda a importância de mais estudos que possam trazer um aprofundamento sobre a temática de Doença Renal Crônica nos diversos ciclos da vida, principalmente quando se trata de jovens.

\section{Referências}

Abreu, N, L, S, et al. Crianças e adolescentes com insuficiência renal em hemodiálise: percepção dos profissionais, Rev. Brasileira de Enfermagem, 68(6), $712-800$.

Mello, M. O protagonismo dos jovens com doença renal crônica e a dádiva na construção da atenção á saúde, Rev. Saúde Soc.,25(1), $206-217$. 
Research, Society and Development, v. 10, n. 12, e236101220101, 2021

(CC BY 4.0) | ISSN 2525-3409 | DOI: http://dx.doi.org/10.33448/rsd-v10i12.20101

Pernnafort, Q. (2011). Componentes clínicos associado aos cuidados de enfermagem a criança e adolescentes com doença renal crônica. Rev. da Rede de Enfermagem do Nordeste, 12(4),.758-766, Universidade Federal do Ceará, Fortaleza, Brasil, 2011.

Rêgo, M, S, et al. Impacto da doença renal crônica em adolescentes em tratamento hemodialítico. Rev. de Enfermagem UFPE On Line, 13 , $240-286$.

Resende, A, C, P, et al. Doença renal crônica e suas consequências na criança e adolescente. Rev. Arch Latin Nefr Ped, 20(1), 40-59.

Silva, L. Implicações sociais do tratamento hemodialítico na vida dos jovens com doença renal crônica, Rev. O Público e O Privado, (31).

Ramos, K., M., R., G., et al. (2018). Humanização na Atenção Primária à Saúde, Rev Med Minas Gerais, 28 (5), p. S280522, [S. L], 2018.

Duarte, H. (2018). A autonomia do paciente com doença renal crônica: percepções do paciente e da equipe de saúde, Rev SBPH, v.21 n.1, Rio de Janeiro, 2018.

Innocencio, C., I., et al. (2017). Resposta emocional de pacientes à terapia com música na hemodiálise: Uma ferramenta de humanização, Rev Arte Méd Ampl. 37 (1), p.5-11, 2017.

Oliveira, S., A., S, C., M., et al. (2016). Qualidade de vida de pacientes em hemodiálise e sua relação com mortalidade, hospitalizações e má adesão ao tratamento, Rev J Bras Nefrol, 38 (4) p. 411-420, [S. 1.], 2016.

Cruz, T., W., et al. (2016). A manutenção da vida laboral por doentes renais crônicos em tratamento de hemodiálise: uma análise dos significados do trabalho, Rev Saúde Soc., 25 (4), p.1050-1063. São Paulo, 2016.

Fernandes, B., O., C., B., et al, (2015). Telemedicina: Desenvolvimento de um sistema para atendimento à distância de pacientes com doença renal crônica pré-dialítica, Rev J Bras Nefrol, 37 (3), p.349-358, [S.L.], 2015.

Pereira, P., A., N., P., B., M., et al, (2016). Prevalência de doença renal crônica em adultos atendidos na Estratégia de Saúde da Família, Rev J Bras Nefrol, 38 (1), p.22-30, [S. L.], 2016.

Balistieri, T., C., et al, (2013). A importância do apoio socio-emocional em adolescentes e adultos jovens portadores de doenças crônica: uma revisão de literatura, Rev Eletrônica Trimestral de Enfermería,30, p.399-409, [S. L.], 2013.

Abralhão, R., A., P., C., A., S., N., L., et al, (2010). Dificuldades vivenciadas pela família e pela criança/ adolescente com doença renal crônica, Rev J Bras Nefrol, 32 (1), p.18-22, [S.L.], 2010.

Damião e A., (2001). A experiência da família ao conviver com a doença crônica da criança. Rev Esc Enf USP, 35 (1) p.66-71, [S.L.] 2001.

Nóbrega, D., R., R., C., (2013). atenção à criança com doença crônica na estratégia saúde da família, Rev Cogitare Enferm., 8 (1), p.57-63, 2013. 